\title{
Exploring the alpha cluster structure of nuclei using the thick target inverse kinematics technique for multiple alpha decays
}

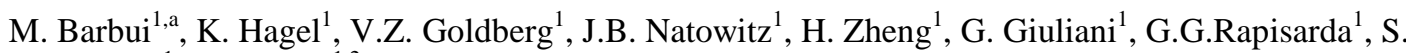 \\ Wuenschel ${ }^{1}$ and X. Liu ${ }^{1,2}$ \\ ${ }^{1}$ Cyclotron Institute, Texas A\&M University, 3366 TAMU, College Station, Texas, USA \\ ${ }^{2}$ Institute of modern physics, Chinese Academy of Sciences, Lanzhou, China
}

\begin{abstract}
We explored alpha clustering in ${ }^{24} \mathrm{Mg}$ using the reaction ${ }^{20} \mathrm{Ne}+\alpha$ and the Thick Target Inverse Kinematics (TTIK) technique. ${ }^{20} \mathrm{Ne}$ beams of energy $3.7 \mathrm{AMeV}$ and 11 $\mathrm{AMeV}$ were delivered by the K150 cyclotron at Texas A\&M University. The reaction chamber was filled with ${ }^{4} \mathrm{He}$ gas at a pressure sufficient to stop the beam before the detectors. The energy of the light reaction products was measured by three silicon detector telescopes. The time relative to the cyclotron radiofrequency was also measured. For the first time the TTIK method was used to study both single and multiple $\alpha$-particle decays. New results were obtained on elastic resonant $\alpha$ scattering, as well as on inelastic processes leading to high excitation energy systems decaying by multiple $\alpha$-particle emission. Preliminary results will be shown on events with $\alpha$-multiplicity one and two.
\end{abstract}

\section{Introduction}

The alpha cluster structure of nuclei with an equal number of protons and neutrons (alpha conjugate nuclei) was categorized in the 1968 by Ikeda et al. [1] to explain some excited states not reproduced by the shell model. Since then many studies have been performed and now alpha clustering in light nuclei is well established [2]. However further investigation is required to fully understand the clusterization in medium light and heavy nuclei. In particular states analogous to the Hoyle state, in which the nucleus is described by a cluster of $\mathrm{n}$ alpha particles, have not yet been unambiguously identified in nuclei larger than ${ }^{12} \mathrm{C}$. In this work we investigated the alpha clustering properties of ${ }^{24} \mathrm{Mg}$ using the reaction ${ }^{20} \mathrm{Ne}+\alpha$ and the Thick Target Inverse Kinematics (TTIK) technique [3]. This technique is particularly suited for this study because it allows the exploration of a large range of incident energies in the same experiment. Moreover, in the inverse kinematics, the reaction products are focused at forward angles and can be detected with detectors covering a relatively small portion of the solid angle in the forward direction.

According to the Ikeda picture [1] ${ }^{24} \mathrm{Mg}$ can be described as ${ }^{20} \mathrm{Ne}+\alpha,{ }^{16} \mathrm{O}+2 \alpha,{ }^{12} \mathrm{C}+3 \alpha$ or a cluster of $6 \alpha$ particles. Each configuration is expected to be observable at excitation energies around the corresponding threshold values. In this experiment the TTIK method [3] was used to study multiple $\alpha$ particle decays as well as elastic scattering. We observed alpha particle multiplicities up to 3 , when the ${ }^{20} \mathrm{Ne}$ beam energy was $2.9 \mathrm{AMeV}$, while at $9.7 \mathrm{AMeV}$ we observed alpha particle multiplicities up to 6 . The results for alpha particle multiplicity one and two are presented here. 


\section{Experimental setup}

${ }^{20} \mathrm{Ne}$ beams of energy 3.7 AMeV and $11 \mathrm{AMeV}$ were delivered by the K150 cyclotron at Texas A\&M University. The effective beam energies after the entrance window were $2.9 \mathrm{AMeV}$ and $9.7 \mathrm{AMeV}$ respectively. The reaction chamber was filled with ${ }^{4} \mathrm{He}$ gas at the pressure of $735 \mathrm{mbar}$ and $3800 \mathrm{mbar}$ respectively, sufficient to stop the beam few centimeters before the detectors. In this way we could detect light particles emitted at zero degrees. The energy of the light reaction products was measured by three silicon detector telescopes placed at a radial distance of $48 \mathrm{~cm}$ from the entrance window. Each telescope consisted of two $5 \times 5 \mathrm{~cm}^{2}$ Micron Semiconductors DC quadrant detectors (Design G). The time of flight of the detected particles was also measured relative to the cyclotron radiofrequency. A monitor detector was used to measure the intensity of the incident beam. A schematic picture of the experimental setup is given in Fig.1.

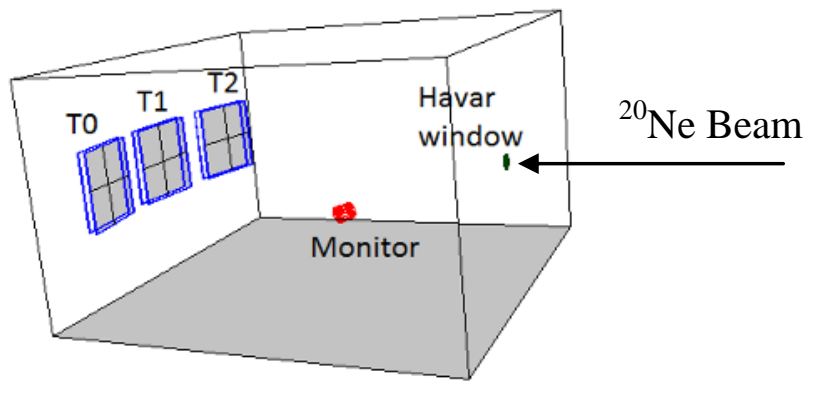

Figure 1. Experimental setup. T0, T1 and T2 are the silicon detector telescopes. The thickness of the Silicon detectors in the telescopes is: T0 (1 mm-2 mm), T1 (1 mm-2 mm), T2 (140 um, $300 \mathrm{um})$.

After the energy and time calibration of the detectors, alpha particles were separated from protons and other light particles with proper gates on $\Delta \mathrm{E}-\mathrm{E}$ and $\mathrm{E}$-Time plots. High $\mathrm{Z}$ reaction products are stopped in the gas before reaching the detectors.

\section{Data Analysis and Results}

A drawback of having a thick target is that we do not have a direct knowledge of the position of the interaction point inside the gas volume and consequently we do not initially know the energy of the beam at that point and the angle of the emitted particle. To overcome this problem, a reconstruction code was developed specifically for this experiment. It allows calculation of the interaction point inside the gas volume, using the reaction kinematics, the measured alpha particle energies and the range energy tables form SRIM [4]. The measured time of flight is also used to double check the position of the interaction point.

Some results obtained on elastic resonant $\alpha$ scattering (alpha multiplicity $=1$ ) are shown in Fig. 2 . The differential cross-sections for resonant elastic scattering, in the center of mass frame, are plotted as a function of the ${ }^{24} \mathrm{Mg}$ excitation energy. With the ${ }^{20} \mathrm{Ne}$ beam at $2.9 \mathrm{AMeV}$ we obtained our best energy resolution $(30 \mathrm{keV})$ at $0^{\circ}$ (worsening away from the beam direction). With the ${ }^{20} \mathrm{Ne}$ beam at 9.7 $\mathrm{AMeV}$ the energy resolution was $60 \mathrm{keV}$ at $0^{\circ}$. The spectra show a very rich structure of levels in ${ }^{24} \mathrm{Mg}$ up to excitation energies of about $30 \mathrm{MeV}$. It is interesting to note that no prominent structures are visible around, $28.5 \mathrm{MeV}$, the energy threshold for the decomposition of ${ }^{24} \mathrm{Mg}$ into 6 alpha particles. In the right panel of Fig. 2 our excitation function at $5^{\circ}$ is compared with that measured by Abegg and Davis [5] at $168^{\circ}$ in normal kinematics, using an alpha particle beam and a $\mathrm{Ne}$ target, in energy steps of 10-15 keV. Even though our resolution is worse than Ref.5, with our technique we could get the gross features of the spectrum in a single run. 

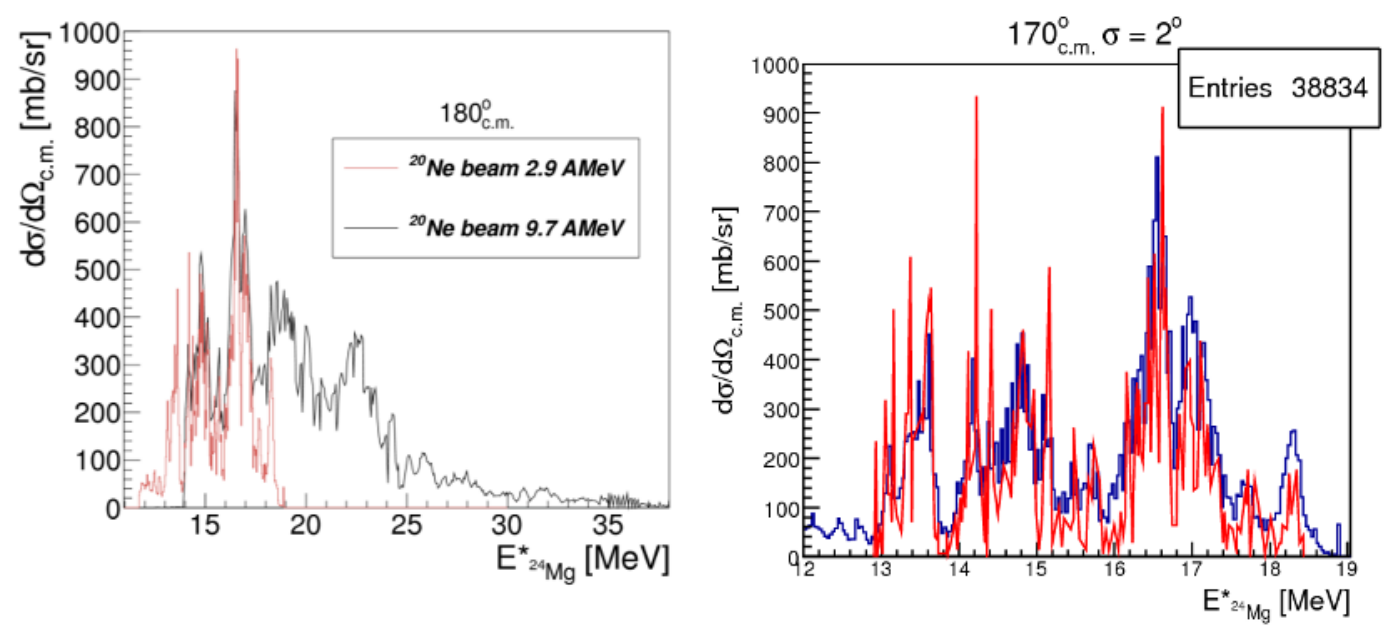

Figure 2. : Resonant elastic scattering. Left panel: Differential cross-sections measured at $0^{\circ}$. Red line beam energy 2.9 AMeV, black line 9.7 AMeV; Right panel: The red line shows the differential cross-section measured in Ref. [5] at $168^{\circ}$ in normal kinematics, the blue line shows our result at $5^{\circ}$ in inverse kinematics.

Events with alpha multiplicity two were detected during both runs. Correlation plots showing the energies of the two detected $\alpha$-particles were produced, as well as similar plots showing the distribution of two uncorrelated events. Uncorrelated events were estimated by randomly mixing two alpha particles from the experimental distribution of multiplicity one events. Figure 3 shows the correlation plots for alpha multiplicity two events, after the subtraction of the uncorrelated events.
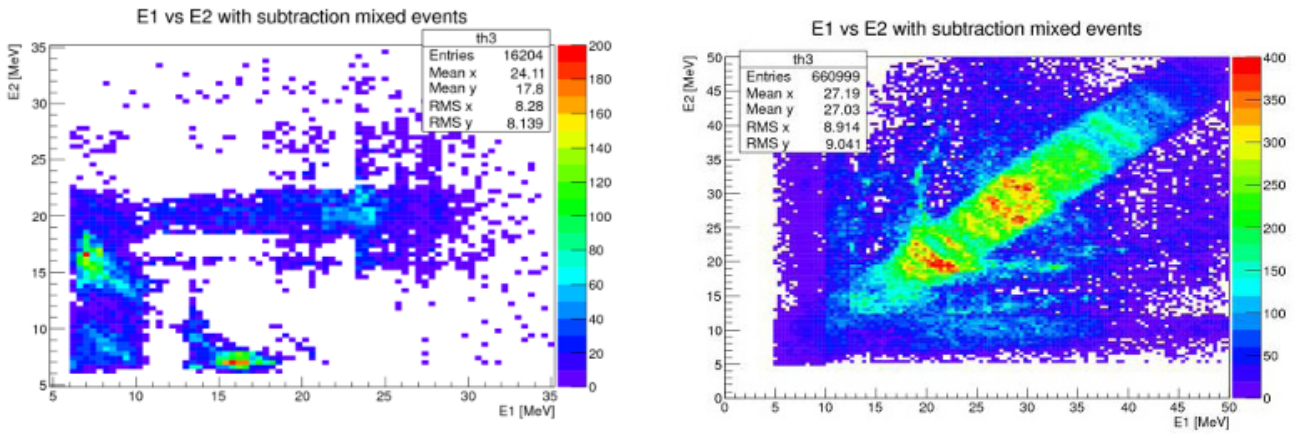

Figure 3. : Energy of the two detected alpha particles in case of $2.9 \mathrm{AMeV}$ (left panel) and $9.7 \mathrm{AMeV}$ beam (right panel).

At the incident energy of 2.9 $\mathrm{AMeV}$ we observed two alpha particles with quite different energies. We made the hypothesis that a resonant alpha scattering populates a ${ }^{24} \mathrm{Mg}$ excited state that have enough energy to emit one alpha particle and leave the ${ }^{20} \mathrm{Ne}$ in an excited state that alpha decays to the ground state of ${ }^{16} \mathrm{O}$. Using this assumption in the reconstruction code we obtained the excitation energy of the ${ }^{24} \mathrm{Mg}$ (from the reconstructed interaction point and the beam energy loss) and the excitation energy of the ${ }^{20} \mathrm{Ne}$ (using the energy of the second alpha particle). The result is shown in Figure 4. It is interesting to note that the excitation energy of ${ }^{24} \mathrm{Mg}$ is in this case very close to the energy threshold to observe the decay into ${ }^{16} \mathrm{O}$ and two alphas.

At the incident energy of $9.7 \mathrm{AMeV}$ we observed several groups of two alpha particles with similar energy emitted mostly at angles close to $0^{\circ}$ in the laboratory frame. We explain these, as alpha particles coming from the splitting of a ${ }^{8} \mathrm{Be}$. Therefore, the hypothesis in the reconstruction of the 
events is that the resonant scattering populates a ${ }^{24} \mathrm{Mg}$ state with enough energy to decay in ${ }^{8} \mathrm{Be}+{ }^{16} \mathrm{O}$. The reconstructed excitation energies of the ${ }^{24} \mathrm{Mg}$ are presented in Figure 5.

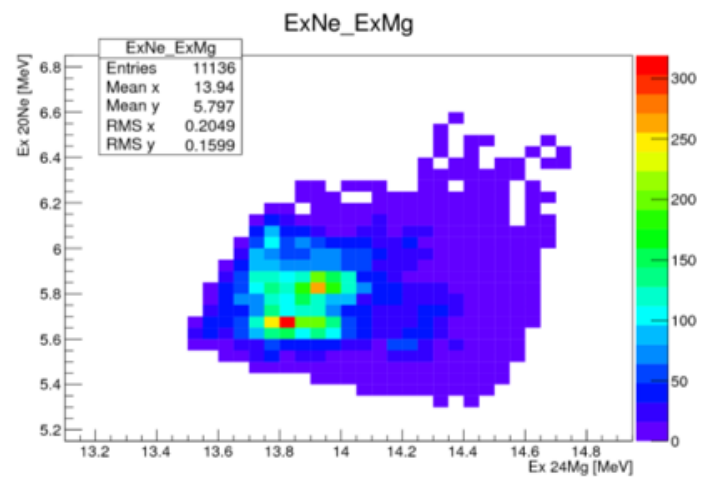

Figure 4. : Reconstructed excitation energies for ${ }^{24} \mathrm{Mg}$ (x-axis) and ${ }^{20} \mathrm{Ne}$ (y-axis). The initial energy of the ${ }^{20} \mathrm{Ne}$ energy was $2.9 \mathrm{AMeV}$.

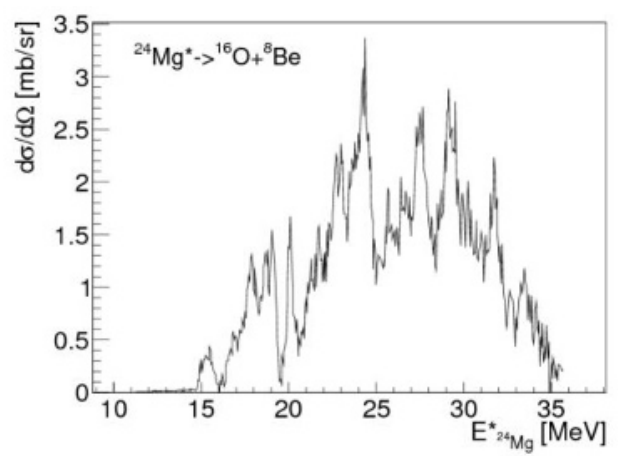

Figure 5. : Reconstructed excitation energy of the ${ }^{24} \mathrm{Mg}$, measured with a ${ }^{20} \mathrm{Ne} 9.7 \mathrm{AMeV}$ at $0^{\circ}$. Very preliminary.

Figure 5 shows a surprisingly high number of resonance peaks in the ${ }^{24} \mathrm{Mg}$ excitation function. These structures might be related with the multiple alpha cluster structure in ${ }^{24} \mathrm{Mg}$. The peaks at about 28 and $32 \mathrm{MeV}$ are particularly interesting because they lie very close to the energy threshold for the splitting of ${ }^{24} \mathrm{Mg}$ into six alphas. Further analysis of the data is necessary to get a final answer.

Another experiment with an improved experimental setup is planned for the near future. A better time resolution is necessary in order to have a better reconstruction of the events. Moreover a larger angular coverage at forward angles is necessary to increase the detection efficiency if we want to be able to improve detection of the events in which the ${ }^{24} \mathrm{Mg}$ decays into six alphas.

\section{References}

1. K. Ikeda, N. Takigawa, and H. Horiuchi, Prog. Theor, Phys. Suppl. Extra Number, 464 (1968)

2. W. von Oertzen, M. Freer, and Y. Kanada-En’yo, Phys. Rep. 432, 43 (2006)

3. K. Artemov et al., Sov. J. Nucl. Phys. 52, 406 (1990)

4. J. F. Ziegler, M. D. Ziegler and J. P. Biersack, Nucl. Instrum. Methods Phys. Res. B 268, 1818 (2010).

5. R. Abegg and C.A. Davis Phys. Rev C 43 (1991) 2523 